\title{
Editorial
}

\section{Intelligent Computational Methods for Financial Engineering}

\author{
Lean Yu, ${ }^{1}$ Shouyang Wang, ${ }^{1}$ and K. K. Lai ${ }^{2}$ \\ ${ }^{1}$ Institute of Systems Science, Academy of Mathematics and Systems Science, \\ Chinese Academy of Sciences, Beijing 100190, China \\ ${ }^{2}$ Department of Management Sciences, City University of Hong Kong, \\ Tat Chee Avenue, Kowloon, Hong Kong \\ Correspondence should be addressed to Lean Yu, yulean@amss.ac.cn
}

Received 17 June 2009; Accepted 17 June 2009

Copyright (C 2009 Lean Yu et al. This is an open access article distributed under the Creative Commons Attribution License, which permits unrestricted use, distribution, and reproduction in any medium, provided the original work is properly cited.

As a multidisciplinary field, financial engineering is becoming increasingly important in today's economic and financial world, especially in areas such as portfolio management, asset valuation and prediction, fraud detection, and credit risk management. For example, in a credit risk context, the recently approved Basel II guidelines advise financial institutions to build comprehensible credit risk models in order to optimize their capital allocation policy. Computational methods are being intensively studied and applied to improve the quality of the financial decisions that need to be made. Until now, computational methods and models are central to the analysis of economic and financial decisions.

However, more and more researchers have found that the financial environment is not ruled by mathematical distributions or statistical models. In such situations, some attempts have also been made to develop financial engineering models using some emerging intelligent computing approaches. For example, an artificial neural network (ANN) is a nonparametric estimation technique which does not make any distributional assumptions regarding the underlying asset. Instead, ANN approach develops a model using sets of unknown parameters and lets the optimization routine seek the best fitting parameters to obtain the desired results. That is, these emerging intelligent solutions can efficiently improve the decisions for financial engineering problems. Having agreed on this basic fact, the guest editors determined that the main purpose of this special issue is not to merely illustrate the superior performance of a new intelligent computational method, but also to demonstrate how it can be used effectively in a financial engineering environment to improve and facilitate financial decision making.

For this purpose, this special issue presents some new progress in intelligent computational methods for financial engineering. In particular, the special issue addresses 
how the emerging intelligent computational methods (e.g., ANN, support vector machines, evolutionary algorithm, and fuzzy models, etc.) can be used to develop intelligent, easy-touse and/or comprehensible computational systems (e.g., decision support systems, agentbased system, and web-based systems, etc.), which is expected to trigger some thoughts and deepen further research.

In this special issue, 12 papers were selected from 26 submissions related to intelligent computational methods for financial engineering from different countries and regions. The authors of the selected papers are from USA, Canada, Australia, Japan, Finland, Tunisia, Sri Lanka, Taiwan, South Africa, and China, respectively. In addition, all the selected papers went through a standard peer review process of the journal and the authors of some papers made necessary revision in terms of reviewing comments. The selected papers include "Optimal Bespoke CDO Design via NSGA-II" by Diresh Jewan, Renkuan Guo, and Gareth Witten, "Modified neural network algorithms for predicting trading signals of stock market indices" by C. D. Tilakaratne, M. A. Mammadov, S. A. Morris, "Selecting the best forecasting implied volatility model using genetic programming" by Wafa Abdelmalek, Sana Ben Hamida, Fathi Abid, "Discrete analysis of portfolio selection with optimal stopping time" by Jianfeng Liang, "A New decision-making method for stock portfolio selection based on computing with linguistic assessment" by Chen Tung Chen, Wei Zhan Hung, "A fuzzy pay-off method for real option valuation" by Mikael Collan, Robert Fuller, Jozsef Mezei, "Valuation for an American continuous-installment put option on bond under vasicek interest rate model" by Guohe Deng, Lihong Huang, "Callable Russian options and their optimal boundaries" by Atsuo Suzuki, Katsushige Sawaki, "Valuation of game options in jump diffusion model and with applications to convertible bonds" by Lei Wang and Zhiming Jin, "Fuzzy real options in brownfield redevelopment evaluation" by Qian Wang, Keith W. Hipel, D. Marc Kilgour, "Discriminant analysis of zero recovery for China's NPL" by Yue Tang, Xiaoguang Yang, Hao Chen, Bo Wang, Muzi Chen, Min Chen, and "Cumulative gains model quality metric" by Thomas Brandenburger, Alfred Furth. The guest editors hope that the papers published in this special issue would be of value to academic researchers and business practitioners and would provide a clearer sense of direction for further research, as well as facilitating use of existing methodologies in a more productive manner.

The guest editors would like to place on record their sincere thanks to Professor Mahyar A. Amouzegar, the Editor-in-Chief of Journal of Applied Mathematics and Decision Sciences, for this very special opportunity provided to us for contributing to this special issue. The guest editors have to thank all the referees for their kind support and help, which has guaranteed that this special issue is of high standard. Finally, the guest editors would like to thank the authors of all the submissions to this special issue for their contribution. Without the support of the authors and the referees, it would have been impossible to make this special issue for our readers. It is hoped that readers can find some topics of interest and benefit to them. The guest editors also hope that this special issue would inspire researchers in the fields of intelligent financial engineering to explore more creative contributions in their research fields.

Lean $Y u$

Shouyang Wang

K. K. Lai 


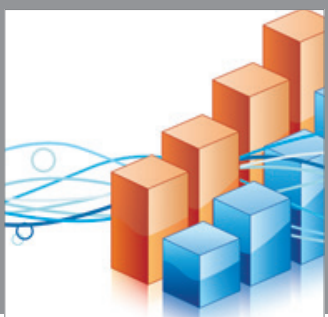

Advances in

Operations Research

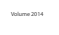

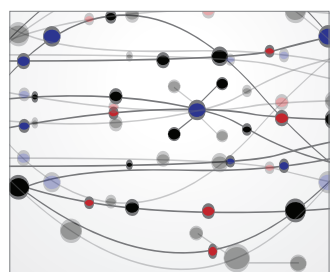

\section{The Scientific} World Journal
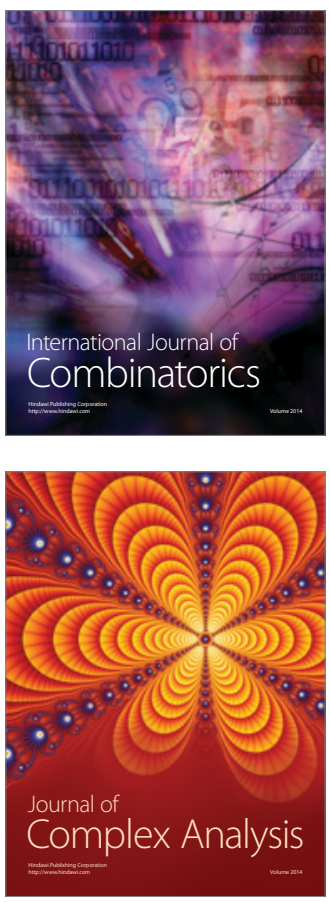

International Journal of

Mathematics and

Mathematical

Sciences
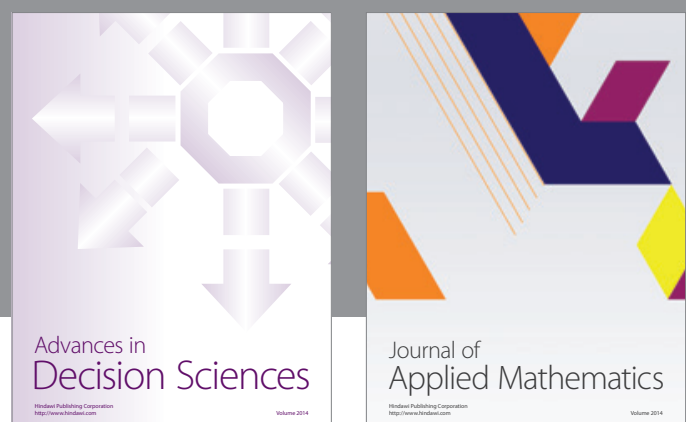

Journal of

Applied Mathematics
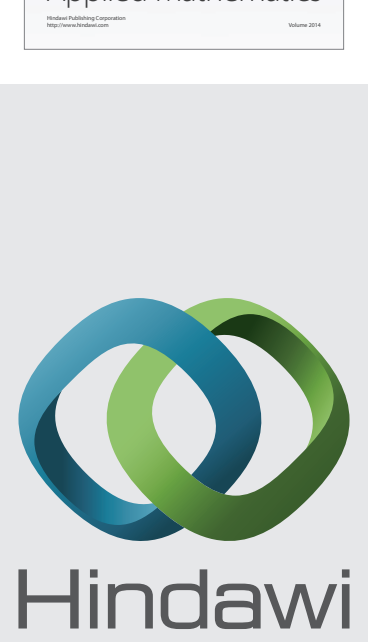

Submit your manuscripts at http://www.hindawi.com
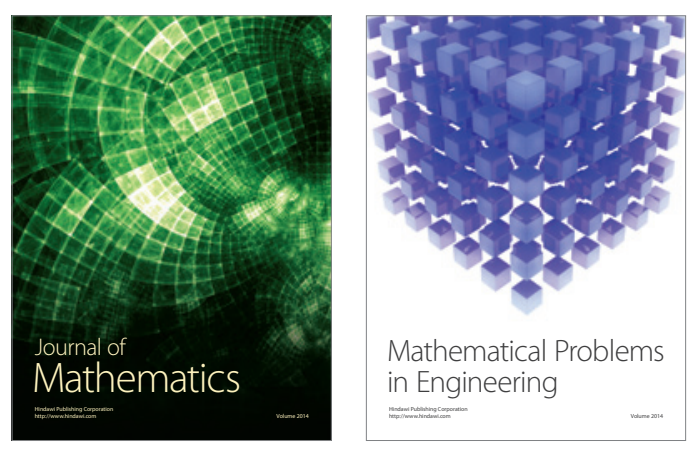

Mathematical Problems in Engineering
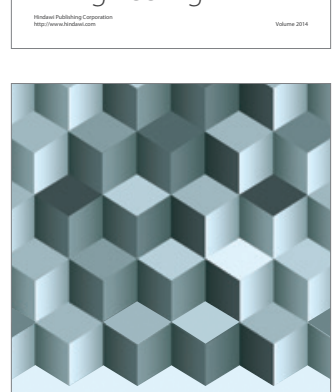

Journal of

Function Spaces
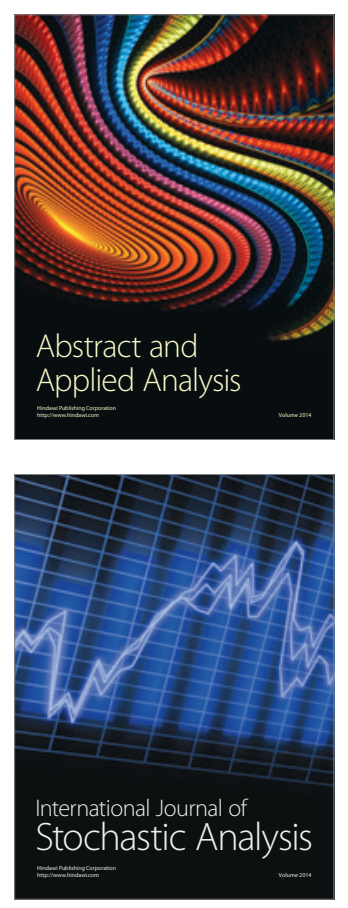

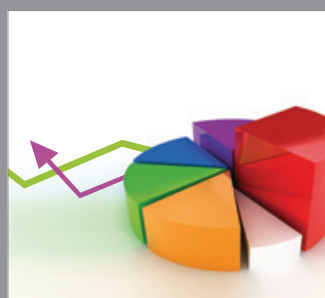

ournal of

Probability and Statistics

Promensencen
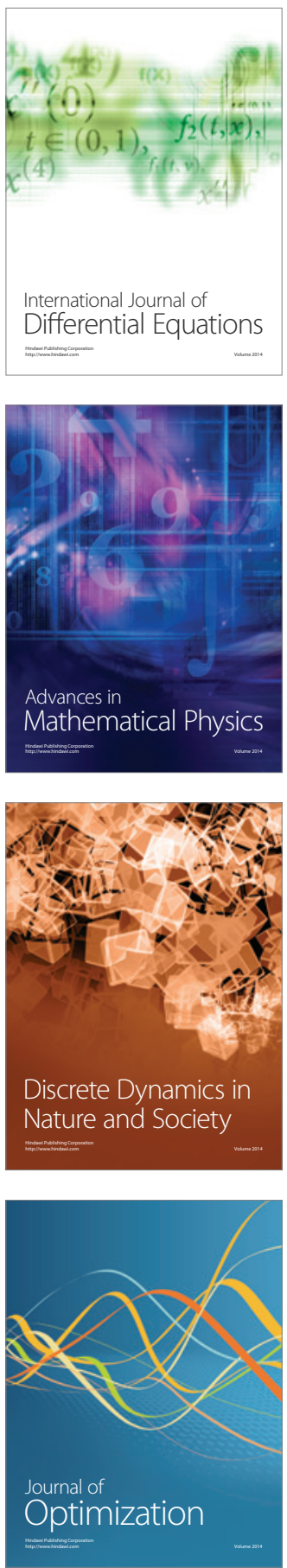